TRANSACTIONS OF THE

AMERICAN MATHEMATICAL SOCIETY

Volume 351, Number 8, Pages 3373-3385

$\mathrm{S}$ 0002-9947(99)02426-5

Article electronically published on April 8, 1999

\title{
DIFFEOMORPHISMS APPROXIMATED BY ANOSOV ON THE 2-TORUS AND THEIR SBR MEASURES
}

\author{
NAOYA SUMI
}

\begin{abstract}
We consider the $C^{2}$ set of $C^{2}$ diffeomorphisms of the 2-torus $\mathbb{T}^{2}$, provided the conditions that the tangent bundle splits into the directed sum $T \mathbb{T}^{2}=E^{s} \oplus E^{u}$ of $D f$-invariant subbundles $E^{s}, E^{u}$ and there is $0<\lambda<1$ such that $\left\|\left.D f\right|_{E^{s}}\right\|<\lambda$ and $\left\|\left.D f\right|_{E^{u}}\right\| \geq 1$. Then we prove that the set is the union of Anosov diffeomorphisms and diffeomorphisms approximated by Anosov, and moreover every diffeomorphism approximated by Anosov in the $C^{2}$ set has no SBR measures. This is related to a result of Hu-Young.
\end{abstract}

We know that Anosov diffeomorphisms are structurally stable (Anosov [A], Robbin [R1] and Robinson [R2]) and have an SBR measure (Sinai [S1]). Recently HuYoung $[\mathrm{H}-\mathrm{Y}]$ showed that a special diffeomorphism $g$ of the 2 -torus $\mathbb{T}^{2}$, provided the condition that for $x \in \mathbb{T}^{2}$ there are $0<\lambda<1$ and a continuous splitting $T_{x} \mathbb{T}^{2}=E_{x}^{u} \oplus E_{x}^{s}$ of invariant subspaces $E_{x}^{u}$ and $E_{x}^{s}$ such that if $x$ is not the origin then (i) $\left\|\left.D g\right|_{E_{x}^{s}}\right\| \leq \lambda$, (ii) $\left\|\left.D g\right|_{E_{x}^{u}}\right\|>1$, and if $p$ is the origin then $\left\|\left.D g\right|_{E_{p}^{u}}\right\|=1$, has no SBR measures. Such a diffeomorphism is called almost Anosov.

Let Diff ${ }^{2}\left(\mathbb{T}^{2}\right)$ be a set of $C^{2}$ diffeomorphisms on the 2-torus imposed with the $C^{2}$ topology. A diffeomorphism $f: \mathbb{T}^{2} \rightarrow \mathbb{T}^{2}$ is called Anosov if $f$ has a hyperbolic structure on all of $\mathbb{T}^{2}$ (cf. [S2]).

We denote as $A\left(\mathbb{T}^{2}\right)$ the open set of Anosov diffeomorphisms. Then $A\left(\mathbb{T}^{2}\right)$ is a proper subset of the set $\theta^{2}$ of diffeomorphisms such that

(iii) the tangent bundle $T \mathbb{T}^{2}$ splits into the directed sum $T \mathbb{T}^{2}=E^{u} \oplus E^{s}$ of invariant subbundles $E^{u}$ and $E^{s}$, and

(iv) there exist a $C^{\infty}$ Riemannian metric $\|\cdot\|$ and $0<\lambda<1$ such that

$$
\left\|\left.D_{x} f\right|_{E^{s}}\right\| \leq \lambda, \quad\left\|\left.D_{x} f\right|_{E^{u}}\right\| \geq 1 .
$$

Our aim is to investigate the dynamical properties of diffeomorphisms belonging to $\theta^{2} \backslash A\left(\mathbb{T}^{2}\right)$. More precisely we state them as follows.

Theorem A. Each diffeomorphism belonging to $\theta^{2} \backslash A\left(\mathbb{T}^{2}\right)$ is approximated by Anosov diffeomorphisms, and it has no SBR measures.

The conclusions will be obtained in proving the following three propositions.

Proposition B. Let $f \in \theta^{2} \backslash A\left(\mathbb{T}^{2}\right)$. Then the set $\Lambda$ defined by

$$
\Lambda=\left\{x \in \mathbb{T}^{2}\left|\left\|\left.D f^{n}\right|_{E_{x}^{u}}\right\|=1 \text { for } n \in \mathbb{Z}\right\}\right.
$$

has the following properties:

Received by the editors February 10, 1997.

1991 Mathematics Subject Classification. Primary 58F11, 58F12, 58F15.

Key words and phrases. Anosov diffeomorphism, SBR measure.

(C)1999 American Mathematical Society 
(a) $\Lambda$ is closed, nonempty and $f$-invariant,

(b) $\Lambda$ is expressed as the union of finite connected sets, and each connected component of $\Lambda$ is either a single point or a $C^{2}$ arc which is tangential to $E^{u}$,

(c) each element of $\Lambda$ is a periodic point, and

(d) there exists a $C^{1}$ Riemannian metric $|\|\cdot \mid\|$ such that

$$
\begin{array}{ll}
|||D f|_{E_{x}^{s}}|| \leq \lambda & \left(x \in \mathbb{T}^{2}\right), \\
|||D f|_{E_{x}^{u}}||>1 & (x \notin \Lambda) \text { and } \\
|||D f|_{E_{x}^{u}}||=1 & (x \in \Lambda) .
\end{array}
$$

By Proposition B we can easily check that the following are equivalent:

(e) $\Lambda$ is a finite set,

(f) $f$ is expansive, i.e. there is a constant $e>0$ such that $x \neq y\left(x, y \in \mathbb{T}^{2}\right)$ implies $d\left(f^{n}(x), f^{n}(y)\right)>e$ for some integer $n$.

By making use of the above metric $\||\cdot|\|$, we have the following:

Proposition C. Every $f \in \theta^{2}$ is $C^{2}$-approximated by Anosov.

Proposition C tells us that every $f \in \theta^{2}$ is homotopic to an Anosov diffeomorphism. Thus there exists a hyperbolic toral automorphism homotopic to $f$, and so $f$ is semi-conjugate to the toral automorphism (see $[\mathrm{A}-\mathrm{H}]$ ). Then we have the following:

Corollary. Let $f \in \theta^{2}$ and let $\Lambda$ be as in Proposition B. Then there exist a hyperbolic toral automorphism $A: \mathbb{T}^{2} \rightarrow \mathbb{T}^{2}$ and a continuous surjective map $h: \mathbb{T}^{2} \rightarrow \mathbb{T}^{2}$ such that

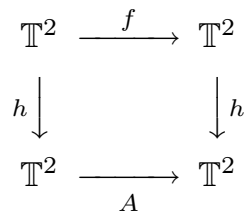

commutes. If $\Lambda$ is finite, then $h$ is a homeomorphism.

The second statement of the corollary follows from the fact that every expansive homeomorphism on the 2-torus is topologically conjugate to a hyperbolic toral automorphism (cf. [H]).

Proposition D. Every $f \in \theta^{2} \backslash A\left(\mathbb{T}^{2}\right)$ has no SBR measures.

For the proof of Proposition D we need the conclusion of Proposition B and the technique in $[\mathrm{H}-\mathrm{Y}]$.

Before starting the proof of Theorem A we give the notations and the definitions that we need. Let $f \in \operatorname{Diff}^{2}\left(\mathbb{T}^{2}\right)$ and $\mu$ be an $f$-invariant Borel probability measure of $\mathbb{T}^{2}$. The measure $\mu$ is called a Sinai-Bowen-Ruelle measure (SBR measure for abbreviation) if for $\mu$-almost all $x \in \mathbb{T}^{2}$ there exist $v \in T_{x} \mathbb{T}^{2}$ and a number $\lambda(x)>0$ satisfying

(A) $\lim _{n \rightarrow \pm \infty} \frac{1}{n} \log \left\|D_{x} f^{n}(v)\right\|=\lambda(x)$,

(B) $\mu$ has a conditional measure that is absolutely continuous (with respect to the Lebesgue measure) on unstable manifolds, which is defined as follows:

If $\xi$ is a measurable decomposition of $\mathbb{T}^{2}$, then a family $\left\{\mu_{x}^{\xi} \mid x \in \mathbb{T}^{2}\right\}$ of Borel probability measures exists, and it satisfies the following conditions: 
(C) for $x, y \in \mathbb{T}^{2}$ if $\xi(x)=\xi(y)$ then $\mu_{x}^{\xi}=\mu_{y}^{\xi}$, here $\xi(x)$ denotes a set containing $x$ in $\xi$,

(D) $\mu_{x}^{\xi}(\xi(x))=1$ for $\mu$-almost all $x \in \mathbb{T}^{2}$,

(E) for any Borel set $A$ a function $x \mapsto \mu_{x}^{\xi}(A)$ is measurable and

$$
\mu(A)=\int_{\mathbb{T}^{2}} \mu_{x}^{\xi}(A) d \mu(x) .
$$

The family $\left\{\mu_{x}^{\xi} \mid x \in \mathbb{T}^{2}\right\}$ is called a canonical system of conditional measures for $\mu$ and $\xi$ (see [R3] for more details).

Whenever $f$ has the condition (A), then a set

$$
W^{u}(x)=\left\{y \in \mathbb{T}^{2} \mid \limsup _{n \rightarrow \infty} \frac{1}{n} \log d\left(f^{-n}(x), f^{-n}(y)\right)<0\right\}
$$

is a unstable manifold for $\mu$-almost all $x$ in $\mathbb{T}^{2}([\mathrm{P}])$. In fact, $W^{u}(x)$ is a $C^{2}$ curve which is homeomorphic to $\mathbb{R}$. A measurable decomposition $\xi$ of $\mathbb{T}^{2}$ is said to be subordinate to unstable manifolds if for $\mu$-almost all $x$ in $\mathbb{T}^{2}$ the following conditions hold:

(F) $\xi(x) \subset W^{u}(x)$,

(G) $\xi(x)$ contains an open arc of $x$ in $W^{u}(x)$.

Let $x \in \mathbb{T}^{2}$ and $m_{x}^{u}$ denote the Lebesgue measure of $W^{u}(x)$. Then a Borel probability measure $\mu$ is called an absolutely continuous conditional measure on unstable manifolds provided the condition that each $\mu_{x}^{\xi}$ in a canonical system of conditional measures is absolutely continuous to $m_{x}^{u}$ for $\mu$-almost all $x$ in $\mathbb{T}^{2}$ if $\xi$ is a measurable decomposition that is subordinate to unstable manifolds. It is known (see [S1], [B], $[\mathrm{L}])$ that every Anosov diffeomorphism has a unique SBR measure.

Proof of Proposition B. It is clear that $\Lambda$ is a $f$-invariant closed set. Thus, to obtain (a) it suffices to show that $\Lambda$ is nonempty. Since $f$ is not Anosov, for $\eta>1$ and $N \geq 1$ there exists $x=x(\eta, N) \in \mathbb{T}^{2}$ such that for $1 \leq n \leq N$

$$
\left\|\left.D f^{n}\right|_{E_{x}^{u}}\right\| \leq \eta .
$$

Indeed, if this is false, then we can find $\eta_{0}>1$ and $N_{0} \geq 1$ such that for $x \in \mathbb{T}^{2}$ there exists $1 \leq n \leq N_{0}$ satisfying $\left\|\left.D f^{n}\right|_{E_{x}^{u}}\right\|>\eta_{0}$. Thus, for $N \geq 1$ and $x \in \mathbb{T}^{2}$ there exist $n_{i}(1 \leq i \leq k)$ and $0 \leq \ell \leq N_{0}-1$ such that $N=n_{1}+n_{2}+\cdots+n_{k}+\ell$ and $\left\|\left.D f^{n_{i+1}}\right|_{E_{x_{i}}^{u}}\right\|>\eta_{0}$ for $0 \leq i \leq k-1$ where

$$
x_{i}= \begin{cases}x & (i=0) \\ f^{n_{1}+n_{2}+\cdots+n_{i}}(x) & (1 \leq i \leq k) .\end{cases}
$$

Since $\left\|\left.D f\right|_{E_{y}^{u}}\right\| \geq 1$ for $y \in \mathbb{T}^{2}$, we have

$$
\begin{aligned}
\left\|\left.D f^{N}\right|_{E_{x}^{u}}\right\| & =\prod_{0}^{k-1}\left\|\left.D f^{n_{i+1}}\right|_{E_{x_{i}}^{u}}\right\|\left\|\left.D f^{\ell}\right|_{E_{x_{k}}^{u}}\right\|>\left(\eta_{0}\right)^{k} \\
& \geq\left(\eta_{0}\right)^{\left[\frac{N}{N_{0}}\right]} \geq\left(\eta_{0}\right)^{\frac{N}{N_{0}}-1}=\left(\eta_{0}\right)^{-1}\left\{\left(\eta_{0}\right)^{\frac{1}{N_{0}}}\right\}^{N} .
\end{aligned}
$$

Put $C=\eta_{0}^{-1}$ and $\eta_{1}=\left(\eta_{0}\right)^{\frac{1}{N_{0}}}$. Since $\eta_{1}$ and $C$ are independent of $x$ in $\mathbb{T}^{2}, f$ is Anosov. This is a contradiction. 
Therefore, for $N \geq 1$ there is $x_{N} \in \mathbb{T}^{2}$ satisfying $1 \leq\left\|\left.D f^{n}\right|_{E_{x_{N}}^{u}}\right\| \leq 1+\frac{1}{N}$ for $1 \leq n \leq 2 N+1$. If $f^{N}\left(x_{N}\right) \rightarrow x_{0}$ (take a subsequence if necessary), then we have that for $i \in \mathbb{Z}$

$$
1 \leq\left\|\left.D f^{i}\right|_{E_{x_{0}}^{u}}\right\|=\lim _{N \rightarrow \infty}\left\|\left.D f^{i}\right|_{E_{f^{N}\left(x_{N}\right)}^{u}}\right\| \leq \lim _{N \rightarrow \infty}\left(1+\frac{1}{N}\right)=1
$$

from which $\Lambda$ is nonempty. (a) is proved.

To show $(\mathrm{d})$, let $\pi: \mathbb{R}^{2} \rightarrow \mathbb{T}^{2}$ denote the natural projection and put $e_{j}=$ $D \pi\left(\frac{\partial}{\partial x_{j}}\right)$ for $j=1,2$. Since $f$ is of $C^{2}$, we remark that for $i \in \mathbb{Z}$ and $j=1,2$, $\varphi_{i, j}(x)=\left\|D_{x} f^{i}\left(e_{j}\right)\right\|$ is a $C^{1}$ function. Choose a sequence $\left\{\delta_{i}\right\}_{i \geq 0}$ of positive numbers satisfying

$$
\sum_{i \in \mathbb{Z}} \delta_{|i|} \max \left\{\varphi_{i, j}(x)^{2},\left\|D_{x} \varphi_{i, j}\right\|^{2} \mid x \in \mathbb{T}^{2}, j=1,2\right\}<\infty,
$$

and define a $C^{1}$ Riemannian metric $\|\mid \cdot\|$ on $T \mathbb{T}^{2}$ by

$$
\||v|\|^{2}=\sum_{i \in \mathbb{Z}} \delta_{|i|}\left\|D_{x} f^{i}(v)\right\|^{2} \quad\left(x \in \mathbb{T}^{2}, v \in T_{x} \mathbb{T}^{2}\right) .
$$

Then it is easily checked that ||$|\cdot|||$ satisfies Proposition B (d).

To show (b) we take a covering map $\bar{\pi}: \mathbb{T}^{2} \rightarrow \mathbb{T}^{2}$ such that $\bar{E}_{x}^{\sigma}=\left(D_{x} \bar{\pi}\right)^{-1} E_{x}^{\sigma}$ $\left(x \in \mathbb{T}^{2}, \sigma=s, u\right)$ is orientable. In fact, $\bar{\pi}$ is 4 to 1 . Let $\bar{f}: \mathbb{T}^{2} \rightarrow \mathbb{T}^{2}$ be a lifting of $f$ by $\bar{\pi}$. Then we have that for $x \in \mathbb{T}^{2}$

$$
\begin{aligned}
D_{x} \bar{f}\left(\bar{E}_{x}^{\sigma}\right) & =\bar{E}_{\bar{f}(x)}^{\sigma} \quad(\sigma=s, u), \\
\left\|\left.D_{x} \bar{f}\right|_{\bar{E}_{x}^{s}}\right\| & \leq \lambda, \\
\left\|\left.D_{x} \bar{f}\right|_{\bar{E}_{x}^{u}}\right\| & \geq 1 .
\end{aligned}
$$

Since $\bar{f}^{2}: \mathbb{T}^{2} \rightarrow \mathbb{T}^{2}$ preserves an orientation of $\bar{E}_{x}^{\sigma}(\sigma=s, u)$, for simplicity we replace $\bar{f}^{2}$ and $\bar{E}^{\sigma}$ by $f$ and $E^{\sigma}$ respectively. Then we can construct a $C^{0}$ vector field $X^{\sigma}: \mathbb{T}^{2} \rightarrow E^{\sigma}(\sigma=s, u)$ which has no singularities.

From the definition of $E^{s}$ it follows that $X^{s}$ is a $C^{1}$ vector field. This is checked by using the ideas in the proof of Theorem 6.3 in [H-P]. Thus a $C^{1}$ foliation of $s$-direction, $\mathcal{F}^{s}$, is constructed. For $x \in \mathbb{T}^{2}$ denote by $W^{s}(x)$ a leaf containing $x$. Then $W^{s}(x)$ is a $C^{2}$ curve which is homeomorphic to $\mathbb{R}$, and has the properties that $T_{y} W^{s}(x)=E_{y}^{s}$ and $d\left(f^{n}(x), f^{n}(y)\right) \rightarrow 0(n \rightarrow \infty)$ for $y \in W^{s}(x)$. Define $W_{\varepsilon}^{s}(x)=\left\{y \in W^{s}(x) \mid d_{s}(x, y) \leq \varepsilon\right\}$ where $d_{s}$ denotes the distance between two points along $W^{s}(x)$. Then we have

$$
W^{s}(x)=\bigcup_{n=0}^{\infty} f^{-n}\left(W_{\varepsilon}^{s}\left(f^{n}(x)\right)\right) \quad\left(x \in \mathbb{T}^{2}\right)
$$

We need a $C^{0}$-foliation of $u$-direction on $\mathbb{T}^{2}$ later. To construct it we must use the splitting

$$
\left\|\left.D f\right|_{E_{x}^{s}}\right\|\left\|\left.D f^{-1}\right|_{E_{f(x)}^{u}}\right\| \leq \lambda \quad\left(x \in \mathbb{T}^{2}\right)
$$

which is called a dominated splitting of $T \mathbb{T}^{2}$. The splitting is obtained from (iv) in the definition of $\theta^{2}$. Though the correspondence $x \mapsto E_{x}^{u}$ is continuous, by the dominated splitting it is ensured (see $[\mathrm{M}])$ that there exists a family $\left\{\tilde{W}_{\varepsilon}^{u}(x) \mid x \in \mathbb{T}^{2}\right\}$ of $C^{2}$ arcs satisfying the conditions:

1. $\tilde{W}_{\varepsilon}^{u}(x) \subset B_{\varepsilon}(x)=\left\{y \in \mathbb{T}^{2} \mid d(x, y) \leq \varepsilon\right\}$ for $x \in \mathbb{T}^{2}$, 
2. $T_{x} \tilde{W}_{\varepsilon}^{u}(x)=E_{x}^{u}$ for $x \in \mathbb{T}^{2}$,

3. letting $\tilde{W}_{\varepsilon^{\prime}}^{u}(x)=B_{\varepsilon^{\prime}}(x) \cap \tilde{W}_{\varepsilon}^{u}(x)$ for $0<\varepsilon^{\prime} \leq \varepsilon$, one can find $\varepsilon^{\prime}>0$ such that

$$
f\left(\tilde{W}_{\varepsilon^{\prime}}^{u}(x)\right) \subset \tilde{W}_{\varepsilon}^{u}(f(x)), \quad f^{-1}\left(\tilde{W}_{\varepsilon^{\prime}}^{u}(x)\right) \subset \tilde{W}_{\varepsilon}^{u}\left(f^{-1}(x)\right),
$$

4. the correspondence $x \mapsto \tilde{W}_{\varepsilon}^{u}(x)$ is continuous with respect to the $C^{2}$ metric. Thus there exists $\delta>0$ such that if $d(x, y)<\delta$ then $W_{\varepsilon}^{s}(x) \cap \tilde{W}_{\varepsilon}^{u}(y)$ is one point and $W_{\varepsilon}^{s}(x)$ is transverse to $\tilde{W}_{\varepsilon}^{u}(y)$. Then we write $[x, y]=W_{\varepsilon}^{s}(x) \cap \tilde{W}_{\varepsilon}^{u}(y)$.

Lemma 1. For $x \in \mathbb{T}^{2}$ the integral curve of $X^{u}$ through $x, \gamma_{x}$, contains the arc $\tilde{W}_{\delta}^{u}(x)$.

Proof. Suppose $\tilde{W}_{\delta}^{u}(x) \not \subset \gamma_{x}$ for some $x \in \mathbb{T}^{2}$. Then we can take $y \in B_{\delta}(x) \cap \gamma_{x}$ such that $y \notin \tilde{W}_{\delta}^{u}(x)$. Thus, $[y, x]=W_{\varepsilon}^{s}(y) \cap \tilde{W}_{\varepsilon}^{u}(x)$. Since $\left\|\left.D f^{-1}\right|_{E^{u}}\right\| \leq 1$, we have $d\left(f^{-1}(y), f^{-1}(x)\right)<\delta$ and then

$$
\left[f^{-1}(y), f^{-1}(x)\right]=W_{\varepsilon}^{s}\left(f^{-1}(y)\right) \cap \tilde{W}_{\varepsilon}^{u}\left(f^{-1}(x)\right)
$$

and thus $f^{-1}([y, x])=\left[f^{-1}(y), f^{-1}(x)\right]$. Repeating this manner, we have

$$
f^{-n}([y, x]) \in W_{\varepsilon}^{s}\left(f^{-n}(y)\right), \quad f^{-n}([y, x]) \neq f^{-n}(y) \quad(n \geq 0) .
$$

Using the fact that $\left\|\left.D f^{-n}\right|_{E^{s}}\right\| \geq \lambda^{-n}$, we have

$$
d_{s}\left(f^{-n}([y, x]), f^{-n}(y)\right) \geq \lambda^{-n} d_{s}([y, x], y) .
$$

This is a contradiction.

Since the integral curve $\gamma_{x}$ is unique, a $C^{0}$ foliation of $u$-direction, $\mathcal{F}^{u}$, is constructed. Denote as $W^{u}(x)$ the leaf containing $x$ in $\mathcal{F}^{u}$. Then it follows that $T_{y} W^{u}(x)=E_{y}^{u}$ and $\tilde{W}_{\varepsilon}^{u}(y) \subset W^{u}(x)$ for $y \in W^{u}(x)$. Remark that each leaf $W^{u}(x)$ is a $C^{2}$ curve.

Lemma 2. For $x, y \in \mathbb{T}^{2}$ the cardinality of $W^{u}(x) \cap W^{s}(y)$ is infinite.

Proof. We first prove the lemma when $W^{u}(x)$ is homeomorphic to $\mathbb{R}$. Since the length of $W^{u}(x), \ell\left(W^{u}(x)\right)$, is infinite, we can find $z_{1}, z_{2} \in W^{u}(x)$ such that $d\left(z_{1}, z_{2}\right)<\delta$ and $\tilde{W}_{\varepsilon}^{u}\left(z_{1}\right) \cap \tilde{W}_{\varepsilon}^{u}\left(z_{2}\right)=\varnothing$.

Since $\left[z_{1}, z_{2}\right]=W_{\varepsilon}^{s}\left(z_{1}\right) \cap \tilde{W}_{\varepsilon}^{u}\left(z_{1}\right) \subset W^{u}(x)$, we denote by $\gamma$ the closed curve which combine the arc in $W^{u}(x)$ from $\left[z_{1}, z_{2}\right]$ to $z_{1}$ with the arc in $W_{\varepsilon}^{s}\left(z_{1}\right)$ from $z_{1}$ to $\left[z_{1}, z_{2}\right]$. We can suppose that $\gamma$ is a Jordan closed curve.

If $\gamma$ is not zero-homotopic, we then obtain the conclusion of Lemma 2. Indeed, let $y \in \mathbb{T}^{2}$. By the assumption, $\mathbb{T}^{2} \backslash \gamma$ is homeomorphic to an annulus. If $W^{s}(y)$ does not intersect to $\gamma$, we then have a contradiction since the existence of a periodic solution of $X^{s}$ in $\mathbb{T}^{2} \backslash \gamma$ is ensured by the Poincaré-Bendixon theorem (Figure 1).

Thus it suffices to prove that $\gamma$ is not zero-homotopic. If it is false, then there exists a 2-disk $D$ in $\mathbb{T}^{2}$ such that the boundary of $D$ is equal to $\gamma$. Since a $C^{1}$ vector field $X^{s}: D \rightarrow E^{s}$ has no singular points, there is a periodic solution in $D$ by the Poincaré-Bendixon theorem. This contradicts the fact that each leaf in $\mathcal{F}^{s}$ is homeomorphic to $\mathbb{R}$.

When $W^{u}(x)$ is a closed curve, we obtain also the conclusion of the lemma.

Lemma 3. For $x \in \mathbb{T}^{2}, \operatorname{cl}\left(W^{u}(x)\right)=\mathbb{T}^{2}$ where $\operatorname{cl}(E)$ denotes the closure of $E$. 


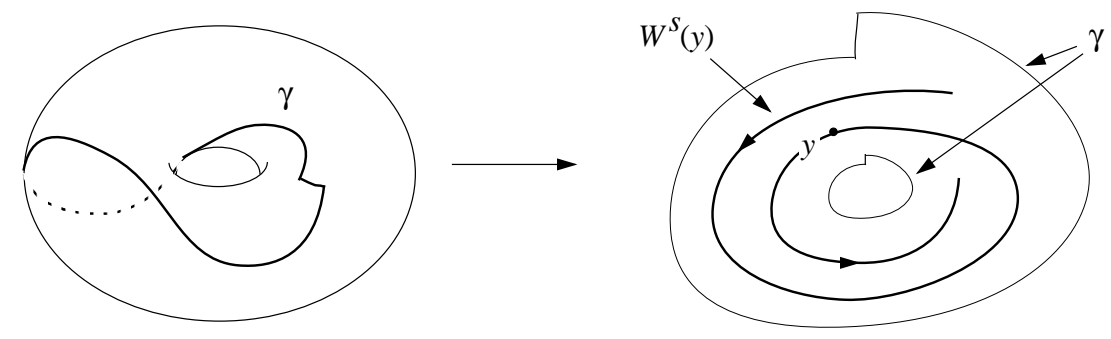

FigURE 1

Proof. We first prove that $W^{u}(x)$ is homeomorphic to $\mathbb{R}$. If this is false, then there exists $z \in \mathbb{T}^{2}$ such that $W^{u}(z)$ is homeomorphic to a circle. Since $\left\|\left.D f^{-1}\right|_{E^{u}}\right\| \leq 1$, we have

$$
\cdots \leq \ell\left(W^{u}\left(f^{-2}(z)\right)\right) \leq \ell\left(W^{u}\left(f^{-1}(z)\right)\right) \leq \ell\left(W^{u}(z)\right) .
$$

Let $\alpha(z)$ denote the set of $\alpha$-limit points of $z$. Take and fix $w \in \alpha(z)$. Then we have $\ell\left(W^{u}\left(f^{n}(w)\right)\right)=\ell\left(W^{u}(w)\right)$ for $n \in \mathbb{Z}$, and so $W^{u}(w)$ is contained in $\Lambda$.

By Lemma 2 we can take $z_{1}, z_{2} \in W^{u}(w)\left(z_{1} \neq z_{2}\right)$ such that $z_{2} \in W^{s}\left(z_{1}\right)$ and $d_{u}\left(z_{1}, z_{2}\right) \leq \delta$ where $d_{u}$ denotes the distance between two points along $W^{u}(w)$. Since $W_{\delta}^{u}\left(z_{1}\right) \subset \Lambda$, we have $f^{n}\left(z_{2}\right) \in W_{\delta}^{u}\left(f^{n}\left(z_{1}\right)\right) \cap W_{\delta}^{s}\left(f^{n}\left(z_{1}\right)\right)$ for some $n>0$, and thus $f^{n}\left(z_{2}\right)=f^{n}\left(z_{1}\right)$. This is a contradiction.

Let $x, y \in \mathbb{T}^{2}$ and let $U$ be a small neighborhood of $y$. To show the density of $W^{u}(x)$ it suffices to see that $U \cap W^{u}(x) \neq \varnothing$. Take a $C^{2}$ arc $I \subset W_{\delta}^{s}(y) \cap U$. Since $\left\|\left.D f^{-1}\right|_{E^{s}}\right\| \geq \lambda^{-1}$, we have

$$
\ell\left(f^{-n}(I)\right) \rightarrow \infty \quad(n \rightarrow \infty)
$$

In a way similar to the proof of Lemma 2 , we have $f^{-n}(I) \cap W^{u}\left(f^{-n}(x)\right) \neq \varnothing$ for some $n>0$ because each leaf of $\mathcal{F}^{u}$ is homeomorphic to $\mathbb{R}$. Therefore, $U \cap W^{u}(x) \supset$ $I \cap W^{u}(x) \neq \varnothing$.

We remark that Lemma 3 is not true for $W^{s}(x)$.

Lemma 4. For $x \in \Lambda$ let $I$ be $a C^{2}$ arc containing $x$ such that $I \subset \tilde{W}_{\delta}^{u}(x)$. If $\ell(I)$ is positive, then

$$
\sum_{n=0}^{\infty} \ell\left(f^{-n}(I)\right)=\infty
$$

Proof. For $\left\{\tilde{W}_{\delta}^{u}(x) \mid x \in \mathbb{T}^{2}\right\}$ a family of $C^{2}$ arcs

$$
\max _{x \in \mathbb{T}^{2}}\left|\frac{d^{2}}{d y^{2}}\left(\left.f\right|_{\tilde{W}_{\delta}^{u}(x)}\right)(y)\right|
$$

is bounded by $K$ from above. Since $f^{-n}(x) \in \Lambda$ and $T_{f^{-n}(x)} \tilde{W}_{\delta}^{u}\left(f^{-n}(x)\right)=E_{f^{-n}(x)}^{u}$ for $n \geq 0$, clearly

$$
\frac{d}{d y}\left(\left.f\right|_{\tilde{W}_{\delta}^{u}\left(f^{-n}(x)\right)}\right)(0)=1
$$


By Taylor's Theorem the graph of $\left.f\right|_{\tilde{W}_{\delta}^{u}\left(f^{-n}(x)\right)}$ satisfies $\left|\left(\left.f\right|_{\tilde{W}_{\delta}^{u}\left(f^{-n}(x)\right)}\right)(y)\right| \leq$ $|F(y)|(|y| \leq \delta, n \geq 0)$ where

$$
F(y)= \begin{cases}y+K y^{2} & (y \geq 0), \\ y-K y^{2} & (y<0)\end{cases}
$$

Thus, $\ell\left(f^{-n}(I)\right) \geq F^{-n}(\delta)$. Since $\sum_{0}^{\infty} F^{-n}(\delta)=\infty$ by ([H-Y] Lemma 4.1), we have $\sum_{0}^{\infty} \ell\left(f^{-n}(I)\right)=\infty$.

Since $\mathcal{F}^{s}$ is a $C^{1}$ foliation of $\mathbb{T}^{2}$, we easily have the following:

Lemma 5. For $x \in \mathbb{T}^{2}$ suppose that $I$ and $J$ are $C^{2}$ arcs contained in $W_{\delta}^{s}(x)$ and $\tilde{W}_{\delta}^{u}(x)$ respectively. Then there exists $\kappa>0$ such that

$$
m([I, J]) \geq \kappa \ell(I) \ell(J)
$$

where $m$ is the Lebesgue measure of $\mathbb{T}^{2}$.

Let $C(x)$ denote the connected component of $x$ in $\Lambda$.

Lemma 6. $C(x) \subset W^{u}(x)$ for $x \in \Lambda$.

Proof. If $C(x) \not \subset W^{u}(x)$ for some $x \in \Lambda$, then there exist $y \in C(x)$ and $\eta>0$ such that $W_{\epsilon}^{u}\left(y^{\prime}\right) \cap \Lambda \neq \varnothing$ for $y^{\prime} \in W_{\eta}^{s}(y)$. Take $z \in \alpha(y)$. Then we have $W^{s}(z) \subset \Lambda$. Since $\left\|\left.D f\right|_{E_{x}^{s}}\right\|<\lambda$ and $\left\|\left.D f\right|_{E_{x}^{u}}\right\|=1$ for $x \in \Lambda$, we have $\Lambda \neq \mathbb{T}^{2}$. Therefore, $\operatorname{cl}\left(W^{s}(z)\right) \neq \mathbb{T}^{2}$.

Fix $w \in \mathbb{T}^{2} \backslash \operatorname{cl}\left(W^{s}(z)\right)$. Let $U$ denote the arcwise connected component of $w$ in $\mathbb{T}^{2} \backslash \operatorname{cl}\left(W^{s}(z)\right)$. Obviously $U$ is open and $f^{-n}(U)$ is the arcwise connected component of $f^{-n}(w)$ in $\mathbb{T}^{2} \backslash \operatorname{cl}\left(W^{s}(z)\right)$ for every $n>0$. Then we have two cases:

(5) $f^{-n}(U) \cap U=\varnothing$ for all $n>0$,

(6) $f^{-n_{0}}(U)=U$ for some $n_{0}>0$.

For (5) we have $\sum_{n=0}^{\infty} m\left(f^{-n}(U)\right)=\infty$. Indeed, from Lemma 3 it follows that the length of the arcwise connected component $I$ of $w$ in $W^{u}(w) \cap U$ is finite. Let $w^{\prime}$ be one of the end points of $I$. Since $U$ is open, $w^{\prime}$ must belong to $\operatorname{cl}\left(W^{s}(z)\right) \subset \Lambda$. Thus Lemmas 4 and 5 ensure that

$$
\begin{aligned}
\sum_{n=0}^{\infty} m\left(f^{-n}(U)\right) & \geq \sum_{n=0}^{\infty} m\left(\left[W_{\delta}^{s}\left(f^{-n}\left(w^{\prime}\right)\right), f^{-n}(I)\right]\right) \\
& \geq \sum_{n=0}^{\infty} \kappa \ell\left(W_{\delta}^{s}\left(f^{-n}\left(w^{\prime}\right)\right)\right) \ell\left(f^{-n}(I)\right) \\
& \geq 2 \kappa \delta \sum_{n=0}^{\infty} \ell\left(f^{-n}(I)\right) \\
& =\infty .
\end{aligned}
$$

When (6) holds, we also have $m(U)=\infty$. Indeed, let $I$ and $w^{\prime}$ be as above. Since $f$ is orientation preserving, we have $f^{-n_{0}}\left(W^{s}\left(w^{\prime}\right)\right)=W^{s}\left(w^{\prime}\right)$. Thus $f^{-n_{0}}$ : $W^{s}\left(w^{\prime}\right) \rightarrow W^{s}\left(w^{\prime}\right)$ is expanding since $\left\|\left.D f^{-1}\right|_{E^{s}}\right\| \geq \lambda^{-1}$, and so there exists a unique fixed point $p \in W^{s}\left(w^{\prime}\right)$ of $f^{-n_{0}}$. Without loss of generality we suppose that $p \neq w^{\prime}$. Then $w^{\prime}$ is not a periodic point. Thus, for $r>0$ small enough we have

$$
\left[W_{r}^{s}\left(f^{-k n_{0}}\left(w^{\prime}\right)\right), f^{-k n_{0}}(I)\right] \cap\left[W_{r}^{s}\left(f^{-k^{\prime} n_{0}}\left(w^{\prime}\right)\right), f^{-k^{\prime} n_{0}}(I)\right]=\varnothing \quad\left(k \neq k^{\prime}\right),
$$


and thus by Lemmas 4 and 5

$$
\begin{aligned}
m(U) & \geq m\left(\bigcup_{k=0}^{\infty}\left[W_{r}^{s}\left(f^{-k n_{0}}\left(w^{\prime}\right)\right), f^{-k n_{0}}(I)\right]\right) \\
& =\sum_{k=0}^{\infty} m\left(\left[W_{r}^{s}\left(f^{-k n_{0}}\left(w^{\prime}\right)\right), f^{-k n_{0}}(I)\right]\right) \\
& \geq \sum_{k=0}^{\infty} \kappa \ell\left(W_{r}^{s}\left(f^{-k n_{0}}\left(w^{\prime}\right)\right)\right) \ell\left(f^{-k n_{0}}(I)\right) \\
& \geq 2 \kappa r \sum_{k=0}^{\infty} \ell\left(f^{-k n_{0}}(I)\right)=\infty .
\end{aligned}
$$

In any case we have $m\left(\mathbb{T}^{2}\right)=\infty$. This is a contradiction.

Lemma 6 tells us that $C(x)$ is either a single point, or a $C^{2}$ arc in $W^{u}(x)$. We remark that $\ell(C(x))$ is finite. This follows from the fact if $\ell(C(x))$ is infinite then $\operatorname{cl}(C(x))=\mathbb{T}^{2}$ by Lemma 3. Therefore the second statement of Proposition B (b) was proved.

Since $\left\|\left.D f\right|_{E_{y}^{u}}\right\|=1$ for $y \in C(x)$, the length of $C(x)$ is $f$-invariant. Then, using the next lemma, it follows that $\left.f^{m(x)}\right|_{C(x)}$ is the identity map of $C(x)$. This implies Proposition B (c).

Lemma 7. For $x \in \Lambda$ there exists $m=m(x)>0$ such that $f^{m}(C(x))=C(x)$.

Proof. We first prove the lemma for the case when $C(x)$ is a $C^{2}$ arc. To see so let $y$ be one of the end points of $C(x)$ in $W^{u}(x)$. It is clear that $\alpha(y) \subset \Lambda$. If we establish that $\alpha(y)$ is finite, then each element belonging to $\alpha(y)$ is periodic. Thus there is $z \in \alpha(y)$ such that $y \in W^{u}(z)$. Then we have $y=z$. Indeed, if $y \neq z$ and $f^{-i}(z)=z$, then we have that for $k>0$

$$
d_{u}(y, z)=d_{u}\left(y, f^{-i}(y)\right)+d_{u}\left(f^{-i}(y), f^{-2 i}(y)\right)+\cdots+d_{u}\left(f^{-k i}(y), z\right) .
$$

Thus we have $d_{u}(y, z)=\infty$ by Lemma 4 since $k$ is arbitrary. This is a contradiction. Therefore $y$ is periodic. Let $m$ be the period of $y$ by $f$. Then $f^{m}(C(x))=C(x)$ since $y$ is an end point of $C(x)$, and since the length of $C(x)$ is $f$-invariant. Thus it suffices to prove that $\alpha(y)$ is finite.

We first prove that $\alpha(y)$ is totally disconnected. If this is false, then there exists $z \in \alpha(y)$ such that $\tilde{W}_{\eta}^{u}(z) \subset \alpha(y)$ for small $\eta>0$. Choose an increasing sequence $\left\{n_{k}\right\}$ such that $\left\{f^{-n_{k}}(y)\right\}$ converges to $z$ as $k \rightarrow \infty$. Then $\left[f^{-n_{k}}(y), z\right]$ converges to $z$ as $k \rightarrow \infty$. Since

$$
\begin{aligned}
d\left(y, f^{n_{k}}(z)\right) & \leq d_{s}\left(y, f^{n_{k}}\left(\left[f^{-n_{k}}(y), z\right]\right)\right)+d_{u}\left(f^{n_{k}}\left(\left[f^{-n_{k}}(y), z\right]\right), f^{n_{k}}(z)\right) \\
& \leq \lambda^{n_{k}} d_{s}\left(f^{-n_{k}}(y),\left[f^{-n_{k}}(y), z\right]\right)+d_{u}\left(\left[f^{-n_{k}}(y), z\right], z\right) \\
& \rightarrow 0 \quad(k \rightarrow \infty),
\end{aligned}
$$

$f^{n_{k}}\left(\tilde{W}_{\eta}^{u}(z)\right)$ converges to $\tilde{W}_{\eta}^{u}(y)$ under the Hausdorff topology. Remark that $f^{n_{k}}\left(\tilde{W}_{\eta}^{u}(z)\right) \subset \Lambda$ for $k>0$ (and then $\tilde{W}_{\eta}^{u}(y) \subset \Lambda$ ). Then we have $\tilde{W}_{\eta}^{u}(y) \subset C(x)$, thus contradicting the choice of $y$. Therefore $\alpha(y)$ is totally disconnected.

Suppose that $\alpha(y)$ is infinite to obtain a contradiction. For $w \in \mathbb{T}^{2}, \tilde{W}_{\epsilon}^{u}(w) \backslash\{w\}$ is expressed as the union $\tilde{W}_{\epsilon}^{u}(w) \backslash\{w\}=I_{w}^{1} \cup I_{w}^{2}$ of $C^{2}$ arcs $I_{w}^{1}$ and $I_{w}^{2}$ in $W^{u}(w)$ 


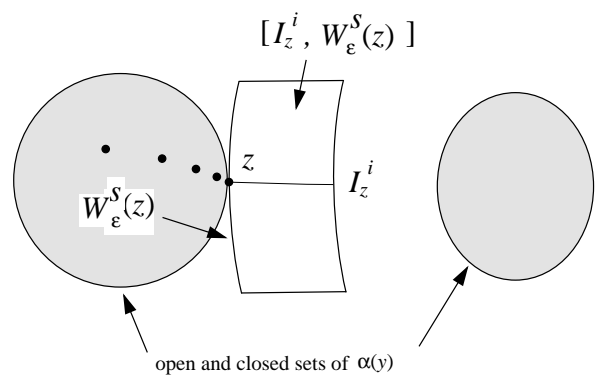

Figure 2

with $I_{w}^{1} \cap I_{w}^{2}=\varnothing$. Since the collection of open closed subsets of $\alpha(y)$ is a base of $\alpha(y)$, there exist a non-periodic point $z \in \alpha(y)$ and $i(=1$ or 2$)$ such that

$$
\operatorname{int}\left(\left[I_{z}^{i}, W_{\epsilon}^{s}(z)\right]\right) \cap \alpha(y)=\varnothing
$$

for $\varepsilon>0$ small enough (Figure 2). Indeed, if $z$ is a periodic point, then we have $y \notin W^{u}(z)$ since $\alpha(y)$ and the orbit of $z$ agree when $y \in W^{u}(z)$, and since $\alpha(y)$ is infinite by the assumption. Thus $\left\{\alpha(y) \cap W^{s}(z)\right\} \backslash\{z\}$ is nonempty, then we can take a non-periodic point from the set. Notice that $f^{-n}(z) \notin W_{\epsilon}^{s}(z)(n>0)$ for sufficiently small $\epsilon>0$. For simplicity put $I_{z}=I_{z}^{i}$.

Take and fix $\tau>0$ small enough. We define $R_{n}=\left[f^{-n}\left(I_{z}\right), W_{\tau}^{s}(z)\right]$ for $n \geq 0$. Then, $R_{n+1} \subset f^{-1}\left(R_{n}\right)$ for all $n \geq 0$, and $R_{n} \cap R_{m}=\varnothing$ for all $n \neq m$. Indeed, suppose $R_{m} \cap R_{n} \neq \varnothing$ for some $m>n \geq 0$. Put $k=m-n$. Since

$$
\varnothing \neq f^{n}\left(R_{m} \cap R_{n}\right) \subset f^{n}\left(f^{-n}\left(R_{k}\right) \cap f^{-n}\left(R_{0}\right)\right)=R_{k} \cap R_{0},
$$

we have that $z \in \operatorname{int}\left(\left[f^{-k}\left(I_{z}\right), W_{\epsilon}^{s}\left(f^{-k}(z)\right)\right]\right)$ since $f^{-k}(z) \notin W_{\epsilon}^{s}(z)$. Therefore, $f^{k}(z) \in \operatorname{int}\left(\left[I_{z}, W_{\epsilon}^{s}(z)\right]\right)$, thus contradicting (7).

Since $R_{n} \cap R_{m}=\varnothing$ for all $n \neq m$, by Lemmas 4 and 5 ,

$$
\begin{aligned}
m\left(\bigcup_{n \geq 0} R_{n}\right) & =\sum_{n \geq 0} m\left(R_{n}\right) \\
& \geq \sum_{n \geq 0} \kappa \ell\left(f^{-n}\left(I_{z}\right)\right) \ell\left(W_{\tau}^{s}(z)\right) \\
& \geq 2 \kappa \tau \sum_{n \geq 0} \ell\left(f^{-n}\left(I_{z}\right)\right) \\
& =\infty .
\end{aligned}
$$

But this is impossible. Therefore $\alpha(x)$ is finite.

When $C(x)$ is a single point, we also obtain the conclusion of the lemma.

To complete the proof of Proposition B it suffices to show that $\Lambda$ splits into the union of finite connected sets. To obtain it suppose the cardinality of $\{C(x) \mid x \in \Lambda\}$ is infinite. If $\left\{x_{i}\right\}$ is an infinite sequence in $\Lambda$ and $x_{i} \rightarrow x$ as $i \rightarrow \infty$, then $x$ is also a periodic point by Proposition B (c).

If $x_{i} \in W^{u}(x)$ for some $i$, then $x$ and $x_{i}$ are joined by a $C^{2} \operatorname{arc} I$ in $W^{u}(x)$. Since $\left\|\left.D f^{-1}\right|_{E^{u}}\right\| \leq 1$, we have $\ell\left(f^{-n}(I)\right) \leq \ell(I)$ for $n>0$. Thus, $\ell\left(f^{n}(I)\right)=\ell(I)$ for all $n \in \mathbb{Z}$ because $x_{i}$ and $x$ are periodic points. This implies that $C\left(x_{i}\right)=C(x)$. 


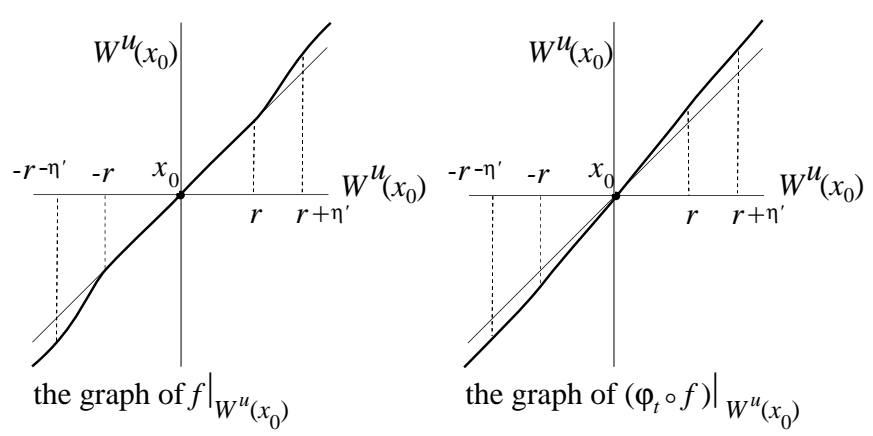

FIGURE 3

Since the cardinality of $\{C(x) \mid x \in \Lambda\}$ is infinite, we may assume that $x_{i} \notin W^{u}(x)$ for all $i$.

Since $x_{i} \notin W^{u}(x)$ for all $i \geq 0$, we can find $y \in W^{s}(x)$ such that $y \in \Lambda$ and $y \neq x$. Then $y \in \Lambda$ is not a periodic point. This contradicts Proposition B (c). Therefore $\Lambda$ is expressed as the union of finite connected sets.

Proof of Proposition C. Let $\Lambda$ be as in Proposition B. We give the proof of Proposition $\mathrm{C}$ for the case when $\Lambda$ is a $C^{2}$ arc. When $\Lambda$ is a single point, or in general, then the conclusion is obtained in a similar argument.

For $x \in \mathbb{T}^{2}$ and $r>0$ we define $W_{r}^{u}(x)=\left\{y \in W^{u}(x) \mid d_{u}(x, y) \leq r\right\}$. Since $\Lambda$ is a $C^{2}$ arc which is tangential to $E^{u}, \Lambda$ is expressed as $\Lambda=W_{r}^{u}\left(x_{0}\right)$ for some $x_{0} \in \Lambda$ and $r>0$. For $\eta>0$ small enough define

$$
R_{\eta}=\bigcup_{x \in W_{r+\eta}^{u}\left(x_{0}\right)} W_{\eta}^{s}(x) .
$$

Since $\Lambda \subset \operatorname{int} R_{\eta}$, by Proposition B (d) there exists $\varepsilon>0$ such that

$$
||\left|D_{x} f\right|_{E^{u}}|| \mid>e^{\varepsilon} \quad\left(x \notin R_{\eta}\right)
$$

where $\left|\left\|\cdot|\||\right.\right.$ is the norm as in Proposition B. A projection $\pi^{u}: R_{\eta} \rightarrow W_{r+\eta}^{u}\left(x_{0}\right)$ defined by $\left\{\pi^{u}(x)\right\}=W_{\eta}^{s}(x) \cap W_{r+\eta}^{u}\left(x_{0}\right)$ for $x \in R_{\eta}$ is a $C^{1}$ map, since $\mathcal{F}^{s}$ is a $C^{1}$ foliation of $\mathbb{T}^{2}$.

Since ||$\left|D_{x} \pi^{u}\right|_{E^{u}} \| \mid=1$ for $x \in \Lambda$, we have that for $0<\eta^{\prime}(<\eta)$ small enough

$$
e^{-\varepsilon / 5} \leq||\left|D_{x} \pi^{u}\right|_{E^{u}}|| \mid \leq e^{\varepsilon / 5} \quad\left(x \in R_{\eta^{\prime}}\right) .
$$

Then we can construct a one-parameter family $\varphi_{t}: \mathbb{T}^{2} \rightarrow \mathbb{T}^{2}(t \in[0,1])$ which satisfies the following:

(10) $\varphi_{t}$ is a $C^{2}$ diffeomorphism for $t \in[0,1]$,

(11) $d_{2}\left(\varphi_{t}\right.$, id $) \rightarrow 0$ as $t \rightarrow 0$ where $d_{2}$ and id denote a $C^{2}$ metric and the identity map respectively,

(12) $\varphi_{t}(x)=x$ for $t \in[0,1]$ and $x \notin R_{\eta^{\prime}}$,

(13) for $t \in(0,1], \varphi_{t}\left(W_{r+\eta^{\prime}}^{u}\left(x_{0}\right)\right)=W_{r+\eta^{\prime}}^{u}\left(x_{0}\right)$ and $\left\|\left|D_{x}\left(\varphi_{t} \circ f\right)\right|_{E^{u}} \mid\right\|>1(x \in$ $\left.W_{r+\eta^{\prime}}^{u}\left(x_{0}\right)\right)$ (Figure 3).

Put $f_{t}=\varphi_{t} \circ f$ for $t \in[0,1]$. Clearly $f_{t}$ is a $C^{2}$ diffeomorphism for $t \in[0,1]$ (by (10)) and $f$ is approximated by $\left\{f_{t}\right\}$ with respect to the $C^{2}$ topology (by (11)). 
The remainder of the proof is only to show that $f_{t}$ is Anosov for small $t \in(0,1]$. By (8) and Proposition B, $f$ satisfies

$$
\begin{cases}||\left|D_{x} f\right|_{E^{s}}|\||||\left|D_{f(x)} f^{-1}\right|_{E^{u}}|| \mid \leq \lambda & \left(x \in \mathbb{T}^{2}\right), \\ ||\left|D_{x} f\right|_{E^{s}} \mid \| \leq \lambda & \left(x \in \mathbb{T}^{2}\right), \\ ||\left|D_{x} f\right|_{E^{u}}|| \mid>e^{\varepsilon} & \left(x \notin R_{\eta}\right), \\ ||\left|D_{x} f\right|_{E^{u}} \mid \|>1 & \left(x \notin R_{\eta^{\prime}}\right) .\end{cases}
$$

Then we can choose $t_{0}>0$ such that for $0<t<t_{0}$ there exist $\lambda<\lambda^{\prime}<1$ and a $D f_{t}$ invariant splitting $T_{x} \mathbb{T}^{2}=E_{x}^{u}(t) \oplus E_{x}^{s}(t)\left(x \in \mathbb{T}^{2}\right)$ satisfying

$$
\begin{cases}\|\left.\left.|| D_{x} f_{t}\right|_{E^{s}(t)}|||||| D_{f(x)} f_{t}^{-1}\right|_{E^{u}(t)}|| \mid \leq \lambda^{\prime} & \left(x \in \mathbb{T}^{2}\right), \\ \|\left|D_{x} f_{t}\right|_{E^{s}(t)}|| \leq \lambda^{\prime} & \left(x \in \mathbb{T}^{2}\right), \\ ||\left|D_{x} f_{t}\right|_{E^{u}(t)} \mid \|>e^{\varepsilon / 2} & \left(x \notin R_{\eta}\right), \\ ||\left|D_{x} f_{t}\right|_{E^{u}(t)} \mid \|>1 & \left(x \notin R_{\eta^{\prime}}\right) .\end{cases}
$$

Fix $t$ with $0<t<t_{0}$. In the same manner as the proof of Proposition B construct a $C^{1}$ foliation $\mathcal{F}_{t}^{s}=\left\{W^{s}(x, t)\right\}_{x \in \mathbb{T}^{2}}$ which is tangential to $E^{s}(t)$. Denote by $W_{\eta}^{s}(x, t)$ the set of $y \in W^{s}(x, t)$ such that $d_{s}(x, y) \leq \eta$, and define a projection $\pi_{t}^{u}: R_{\eta^{\prime}} \rightarrow$ $W_{r+\eta}^{u}\left(x_{0}\right)$ by

$$
\left\{\pi_{t}^{u}(x)\right\}=W_{\eta}^{s}(x, t) \cap W_{r+\eta}^{u}\left(x_{0}\right) \quad\left(x \in R_{\eta^{\prime}}\right) .
$$

By (9) we can assume that for every $x \in R_{\eta^{\prime}}$

$$
e^{-\varepsilon / 4} \leq||\left|D_{x} \pi_{t}^{u}\right|_{E^{u}(t)}|| \mid \leq e^{\varepsilon / 4}
$$

(by taking $t_{0}$ sufficiently small if necessary).

Put $\mu_{t}=\inf \left\{||\left|D_{x} f_{t}\right|_{E^{u}(t)}|||| x \in W_{r+\eta}^{u}\left(x_{0}\right)\right\}$. Then, $\mu_{t}>1$ by (12) and (13). If $f^{i}(x) \in R_{\eta^{\prime}}$ for $0 \leq i \leq n$, then we have ||$\left|D_{x} f_{t}^{n}\right|_{E^{u}(t)} \mid \| \geq e^{-\varepsilon / 2} \mu_{t}^{n}$. This follows from the fact that by (14)

$$
\begin{aligned}
||\left|D_{x} f_{t}^{n}\right|_{E^{u}(t)}|| \mid & \geq\left.||\left|\left(\left.D_{f_{t}^{n}(x)} \pi_{t}^{u}\right|_{E^{u}(t)}\right)^{-1}\right|||||\left|D_{\pi_{t}^{u}(x)} f_{t}^{n}\right|_{E^{u}(t)}|||||| D_{x} \pi_{t}^{u}\right|_{E^{u}(t)}|| \mid \\
& \geq e^{-\varepsilon / 4} \mu_{t}^{n} e^{-\varepsilon / 4}=e^{-\varepsilon / 2} \mu_{t}^{n} .
\end{aligned}
$$

Let $K_{1}$ be a large number such that for $x \in R_{\eta^{\prime}}$ and $g\left(=f\right.$ or $\left.f^{-1}\right)$ if $g(x) \notin R_{\eta^{\prime}}$ then $g^{K_{1}}(x) \notin R_{\eta}$, and $K_{2}$ be a positive integer satisfying $e^{-\varepsilon / 2} \cdot \mu_{t}^{K_{2}}>1$. Put $K=2 K_{1}+K_{2}$. Then we have $\left\|\left|D_{x} f_{t}^{K}\right|_{E^{u}(t)}\right\| \mid>1$ for $x \in \mathbb{T}^{2}$. Therefore it follows that $f_{t}$ is Anosov by the technique of the proof of Proposition B (a).

Proof of Proposition D. For the proof we need Proposition B and the technique used in $[\mathrm{L}-\mathrm{Y}]$. Suppose the proposition is false. For $\varepsilon>0$ we have (see [L-Y], Lemma 3.2 and Corollary 6.2) that there is a Borel set $S$ such that the following conditions hold:

(15) $\mu(S)>0$.

(16) There is a family $\left\{D_{\alpha}\right\}$ of $C^{2}$ arcs satisfying

(16-1) $D_{\alpha} \cap D_{\alpha^{\prime}}=\varnothing \quad\left(\alpha \neq \alpha^{\prime}\right)$,

(16-2) $S=\bigcup_{\alpha} D_{\alpha}$,

(16-3) if $x \in D_{\alpha}$, then $D_{\alpha} \subset \tilde{W}_{\varepsilon}^{u}(x)$ and $D_{\alpha}$ is open in $\tilde{W}_{\varepsilon}^{u}(x)$.

(17) Let $\left\{\mu_{\alpha}\right\}$ be a canonical system of conditional Borel probability measures, then each $\mu_{\alpha}$ is absolutely continuous with respect to the Lebesgue measure 
$m_{\alpha}$ of $D_{\alpha}$, and if $\rho_{\alpha}: D_{\alpha} \rightarrow \mathbb{R}^{+}$is the density function of $m_{\alpha}$, then there is $L>0$ such that for $(x, y) \in D_{\alpha} \times D_{\alpha}$

$$
\left|\log \frac{\rho_{\alpha}(y)}{\rho_{\alpha}(x)}\right| \leq L d_{u}(x, y)
$$

By (16-3) and (17) we have

$$
\left|\frac{\rho_{\alpha}(x)}{\rho_{\alpha}(y)}\right| \leq e^{L \varepsilon}
$$

Since $\mu$ is a SBR measure of $\mathbb{T}^{2}$ by the assumption, $\mu$-almost all $x$ has a positive Lyapunov exponent. Then, for $\mu$-a.e. $x, E_{x}^{u}$ is the subspace corresponding to the exponent. Since $f \in \theta^{2} \backslash A\left(\mathbb{T}^{2}\right)$, we have $\left\|\left.D f\right|_{E^{u}}\right\| \geq 1$ and thus $\ell\left(f^{n} D_{\alpha}\right) \geq \ell\left(D_{\alpha}\right)$. If a point in $D_{\alpha}$ has a positive Lyapunov exponent, then $\ell\left(f^{n} D_{\alpha}\right) \nearrow \infty$ as $n \rightarrow \infty$. Therefore, without loss of generality we can suppose that $\ell\left(f^{n} D_{\alpha}\right) \nearrow \infty$ for all $\alpha$.

Let $\Lambda$ be as in Proposition B and take $z \in \Lambda$. By Proposition B, $z$ is a periodic point of $f$. For simplicity we assume that $z$ is a fixed point. By Lemma 3 there exists $n(\alpha)=n \geq 0$ such that

$$
f^{n}\left(D_{\alpha}\right) \cap W^{s}(z) \neq \varnothing
$$

Thus we have

$$
D_{\alpha} \cap W^{s}(z)=f^{-n}\left(f^{n}\left(D_{\alpha}\right)\right) \cap W^{s}(z) \neq \varnothing .
$$

Since $D_{\alpha}$ contains a point with the positive Lyapunov exponent, we can find a compact subset $C \subset W^{s}(z)$ such that

$$
\mu\left(\bigcup_{\alpha}\left\{D_{\alpha} \mid D_{\alpha} \cap C \neq \varnothing\right\}\right)>0 .
$$

Choose $k>0$ such that $d_{s}\left(f^{k}(y), z\right)$ is small enough for all $y \in C$, and put $C^{\prime}=f^{k}(C)$ for simplicity. Without loss of generality we can suppose

$$
\left[\tilde{W}_{\tau}^{u}(z), C^{\prime}\right] \subset \bigcup_{\alpha} f^{k}\left(D_{\alpha}\right)
$$

for $\tau>0$ small enough. Define $R_{n}=\left[\tilde{W}_{\tau}^{u}(z), f^{n}\left(C^{\prime}\right)\right]$ for $n \geq 0$. Then we have that for $n \neq m, R_{n} \cap R_{m}=\varnothing$ by taking the size of $C^{\prime}$ sufficiently small. We remark that there is $\eta>0$ such that for $y \in \tilde{W}_{\tau}^{u}(z)$ and $w \in C^{\prime}$

$$
d_{u}([y, w], w) \geq \eta d_{u}(y, z)
$$

since $\mathcal{F}^{s}$ is a $C^{1}$ foliation of $\mathbb{T}^{2}$.

Put $S_{0}=\bigcup_{\alpha} f^{k}\left(D_{\alpha}\right)$. Then $S_{0}$ is a Borel set satisfying the conditions (15), (16) and (17). Thus the $\mu$-values of $\left[\tilde{W}_{\tau}^{u}(z), C^{\prime}\right]$ are positive by (18), (19) and (20). Since $\left\{\left[\tilde{W}_{\tau}^{u}(z), y\right] \mid y \in C^{\prime}\right\}$ is a decomposition of $S_{1}=\left[\tilde{W}_{\tau}^{u}(z), C^{\prime}\right], S_{1}$ is a Borel set 
satisfying (15), (16) and (17). Thus we have that

$$
\begin{aligned}
\sum_{n} \mu\left(R_{n}\right) & =\sum_{n} \mu\left(f^{-n}\left(R_{n}\right)\right) \\
& =\sum_{n} \mu\left(f^{-n}\left(\left[\tilde{W}_{\tau}^{u}(z), f^{n}\left(C^{\prime}\right)\right]\right)\right) \\
& =\sum_{n} \mu\left(\left[f^{-n}\left(\tilde{W}_{\tau}^{u}(z)\right), C^{\prime}\right]\right) \\
& \geq \sum_{n} e^{-L \varepsilon} \eta \ell\left(f^{-n}\left(\tilde{W}_{\tau}^{u}(z)\right)\right) \quad(\text { by }(18) \text { and }(21)) .
\end{aligned}
$$

Since $\mu\left(R_{1}\right)>0$, by applying Lemma 4 we have $\mu\left(\bigcup R_{n}\right)=\infty$. This is a contradiction.

\section{REFERENCES}

[A] D.V. Anosov, Geodesic flows on closed Riemannian manifolds with negative curvature, Proc. Steklov Inst. Math. 90 (1967), 1-235. MR 39:3527

[A-H] N. Aoki and K. Hiraide, Topological Theory of Dynamical Systems, Mathematical Library, North-Holland, 1994. MR 95m:58095

[B] R. Bowen, Equilibrium States and the Ergodic Theory of Anosov Diffeomorphisms, Lecture Notes in Math. 470, Springer-Verlag, 1975. MR 56:1364

$[\mathrm{H}] \quad$ K. Hiraide, Expansive homeomorphisms of compact surfaces are pseudo-Anosov, Osaka J. Math. 27 (1990), 117-162. MR 91b:58184

[H-Y] H. Hu and L.S. Young, Nonexistence of SBR measures for some diffeomorphisms that are 'Almost Anosov', Ergod. Th. \& Dynam. Sys. 15 (1995), 67-76. MR 95j:58096

[H-P] M. Hirsch and C. Pugh, Stable manifolds and hyperbolic sets, Global Analysis, Proc. Sympos. Pure Math. 14, Amer. Math. Soc. (1970), 133-163. MR 42:6872

[L] F. Ledrappier, Propriétés ergodiques des measures de Sinai, Publ. Math. I.H.E.S. 59 (1984), 163-188. MR 86f:58092

[L-Y] R. Ledrappier and L.S. Young, The metric entropy of diffeomorphisms I, Ann. of Math. 122 (1985), 509-539. MR 87i:58101a

[M] R. Mañé, Contributions to the stability conjecture, Topology 17 (1978), 383-396. MR 84b:58061

[P] Y.B. Pesin, Families of invariant manifolds corresponding to non-zero characteristic exponents, Math. USSR Izvestija 10 (1978), 1261-1305. MR 56:16690

[R1] J. Robbin, A structural stability theorem, Ann. of Math. 94 (1971), 447-493. MR 44:4783

[R2] C. Robinson, Stability theorems and hyperbolicity in dynamical systems, Rocky Mountain J. Math. 7 (1977), 425-437. MR 58:13200

[R3] V.A. Rohlin, Lectures on the theory of entropy of translations with invariant measures, Russian Math. Surveys 22:5 (1967), 1-54. MR 36:349

[S1] Ya.G. Sinai, Gibbs measures in ergodic theory, Russ. Math. Surveys 166 (1972), 21-69. MR 53:3265

[S2] S. Smale, Differentiable dynamical systems, Bull. Amer. Math. Soc. 73 (1967), 747-817. MR 37:3598

Department of Mathematics, Tokyo Metropolitan University, Minami-Ohsawa 1-1, HACHIOJI, TOKYO 192-03, JAPAN

E-mail address: sumi@math.metro-u.ac.jp 